\title{
Modifier genes in hypertrophic cardiomyopathy patients of south Indian cohort
}

\author{
Advithi Rangaraju ${ }^{1, ~}$, Satyanarayana Matsa.Lova ${ }^{1}$, Narasimhan Calambur ${ }^{2}$, Pratibha Nallari ${ }^{1}$ \\ ${ }^{1}$ Department of Genetics, Osmania University, Jamia Osmania, Hyderabad, Andhra Pradesh \\ ${ }^{2}$ Cardiologist, CARE Hospitals, Hyderabad, Andhra Pradesh
}

\section{Email address:}

prathinallari@yahoo.com (P. Nallari), advithi.rangaraju@gmail.com (A. Rangaraju)

\section{To cite this article:}

Advithi Rangaraju, Satyanarayana Matsa.Lova, Narasimhan Calambur, Pratibha Nallari. Modifier Genes in Hypertrophic Cardiomyopathy Patients of South Indian Cohort. International Journal of Genetics and Genomics. Vol. 2, No. 5, 2014, pp. 84-91.

doi: 10.11648/j.ijgg.20140205.12

\begin{abstract}
Hypertrophic cardiomyopathy is an autosomal dominant disorder, characterized by thickening of the myocardium with a variable clinical course. Mutations in 14 sarcomeric genes have been implicated resulting in phenotypic and genotypic heterogeneity. The phenotypic expression of HCM is not only determined by the sarcomeric gene mutations but the genetic predilection of an individual also account for the inter-individual variability and genes with such functional variants that affect phenotypic expression are referred to as Modifier genes. Hence genetic variants of Angiotensin converting enzyme $(A C E-2)$, Tumor necrosis factor- alpha $(T N F-\alpha)$ and Heat shock protein -70 (HSP70) genes have been considered in the present study to understand their role as modifiers of HCM. The study was carried out by Genotyping of 100 HCM and 100 controls by Polymerase chain reaction based restriction fragment length polymorphism analysis. The present study revealed a significant association of the HSP70-1 and HSP70-2 polymorphisms while $A C E-2$ and $T N F-\alpha$ genes were found to be statistically insignificant. However patients with the rare/variant genotypes were observed to have stronger clinical manifestations and echocardiographic parameters. This was further confirmed by Linkage disquillibrium analysis wherein individuals with the haplotypes GCGC and GCGT seemed to have increased susceptibility to HCM. The MDR analysis revealed a synergistic interaction of $T N F-\alpha$ with $A C E-2$ and $H S P 70$ polymorphisms indicating their modifying effect in the presence of other environmental factors. Hence the present study emphasized the role of $A C E-2, T N F-\alpha$ and $H S P 70$ polymorphisms as modifiers of the phenotypic expression in conjunction with other sarcoemric mutations and single nucleotide variations.
\end{abstract}

Keywords: Hypertrophic Cardiomyopathy, Heat Shock Protein-70, Modifier Genes, Angiotensin Converting Enzyme-2, Tumor Necrosis Factor-Alpha, Linkage Disequilibrium

\section{Introduction}

Hypertrophic cardiomyopathy (HCM) is a disease of the myocardium, clinically diagnosed by the presence of left ventricular hypertrophy in the absence of an increased external load and pathologically by the presence of myocyte hypertrophy, disarray and interstitial fibrosis. It has an autosomal dominant mode of inheritance with a prevalence of 1 in 500 exhibiting variable clinical manifestations ranging from an asymptomatic course to severe heart failure and sudden cardiac death (SCD) accounting to genetic heterogeneity [1]. Modifier genes are known to influence the expression and manifestation of the disease. In the presence of causal mutations, they affect the severity of phenotypes, such as the magnitude of cardiac hypertrophy and the risk to $\mathrm{SCD}$ accounting to phenotypic heterogeneity. Hence the final phenotype is the result of the interaction between the causal genes, genetic background (modifier genes), and the environmental factors which account for the inter-individual variation [2].

Identification of the modifier genes will complement the results of studies on causative genes and could enhance genetic based diagnosis, risk stratification, and implementation of preventive and therapeutic measures in patients with HCM. Therefore, in the present study genetic variants of Angiotensin converting enzyme-2 (ACE-2), Tumor Necrosis Factor- $\alpha(T N F-\alpha)$ and Heat shock protein-70 (HSP70) genes have been considered, to understand their role as modifiers of HCM. 


\subsection{Angiotensin Converting Enzyme-2 (ACE-2)}

Genes within the renin-angiotensin-aldosterone system (RAAS) have been investigated as modifiers of the hypertrophy in HCM. Their role in controlling blood pressure and biological effects on cardiomyocyte hypertrophy lay emphasis on the possible role of the angiotensin-convertingenzyme (ACE) in the development of hypertrophy [2,3]. The subsequent discovery of $A C E-2$ and its product Angiotensin (1-7), has been found to antagonize the vasoconstriction mediated by its substrate, Angiotensin II, which is homologous to ACE, sharing about $42 \%$ amino acid sequence identity to the catalytic domain of ACE with 18 exons, encoding a 805 amino-acid polypeptide and mapped onto the Xp22 region [4,5].

ACE2 plays a role as a modifier of hypertrophy in HCM, independent of the effect of the disease-causing mutations suggesting to be an important determinant of left ventricular hypertrophy in HCM patients. Various polymorphisms in $A C E-2$ gene have been studied viz: rs1978124, rs2285666, rs879922, rs4646179, rs4646156, rs4240157 and rs233575, among which rs2106809 has been reported to cause left ventricular hypertrophy in a Chinese cohort [6]. Therefore, due to the genetic diversity of Indian population, $A C E-2$ polymorphism (rs2106809) was studied to understand its role as modifiers of HCM phenotype.

\subsection{Tumor Necrosis Factor- $\alpha-(T N F-\alpha)$}

$T N F-\alpha$ is a pleiotropic cytokine produced mainly by activated monocytes/macrophages and provokes a hypertrophic response in cardiomyocytes on exposure to an environmental stress. The $T N F-\alpha$ gene has a $12 \mathrm{~kb}$ genomic DNA, mapped to $6 \mathrm{p} 21.3$ region of MHC class III and comprises of 4 exons with several polymorphic sites, including microsatellites and promoter polymorphisms [7]. Animal studies have shown that over-expression or chronic infusion of $T N F-\alpha$ can lead to myocarditis, systolic dysfunction, ventricular dilatation, hypertrophy, myocardial fibrosis, myocyte apoptosis, and increased mortality [8-10]. The promoter polymorphisms $-238 \mathrm{G} / \mathrm{A}$ and $-308 \mathrm{G} / \mathrm{A}$ have been widely studied in various diseases [11]. Therefore, the present study considered genotyping of $-308 \mathrm{G} / \mathrm{A}$ polymorphism where the presence of $G$ allele defines the wild type and ' $A$ ' defines rare/ variant allele which is a more powerful transcriptional activator than the wild type allele [12]. Hence, this polymorphism has been studied to elucidate its role as modifiers of HCM.

\subsection{Heat Shock Protein -70 (HSP-70)}

HSP-70 gene, located in the class III region of the major histocompatibility complex (MHC) on 6p21.3, are expressed in response to heat shock and a variety of other stress stimuli and also act as molecular chaperones involved in protein folding, translocation, or degradation and in the regulation of transcription factors and protein kinases [13].

Three polymorphisms of HSP70 gene have been studied viz: HSP 70-1(+190G/C); HSP 70-2(+1267A/G); and HSP70-hom $(+2437 \mathrm{C} / \mathrm{T})$. The HSP 70-1(+190G/C) polymorphism is a silent substitution which lies 26 bp upstream of the translation start site in the 5'UTR region. The $+1267 \mathrm{~A} / \mathrm{G}$ polymorphism of HSP 70-2 lies in the coding region of the gene. The HSP-70-hom $+2437 \mathrm{C} / \mathrm{T}$ results in a missense substitution Met493Thr, which may affect the substrate specificity and chaperone activity and could be associated with variation in peptide binding specificity of HSP70-hom.

The present study revealed a significant association of the HSP70-1 and HSP70-2 polymorphisms while ACE-2 and $T N F-\alpha$ genes were found to be statistically insignificant. However patients with the variant genotypes were observed to have stronger clinical manifestations and echocardiographic parameters. This was further confirmed by Linkage disquillibrium analysis wherein individuals with the haplotypes GCGC and GCGT seemed to have increased susceptibility to HCM. The MDR analysis revealed a synergistic interaction of $T N F-\alpha$ with $A C E-2$ and $H S P 70$ polymorphisms indicating their modifying effect in the presence of other environmental factors.

\section{Clinical Evaluation}

HCM was diagnosed by Physical examination, Electrocardiogram (ECG) and Echocardiography as referred by the consultant cardiologists at CARE Hospitals Nampally, Banjara Hills and Secunderabad. 100 unrelated probands (76 males and 24 females) with a mean age of $45.5 \pm 13.8$ yrs and $41.2 \pm 20.2 \mathrm{yrs}$ in males and females respectively were considered. 100 healthy control samples (60 males and 40 females) were also included in the study after taking the informed written consent and Institutional ethical clearance (Table-1).

Table 1. Epidemiological characteristics of HCM patients

\begin{tabular}{llclc}
\hline \multirow{2}{*}{ Parameter } & Males & \multicolumn{3}{l}{ Females } \\
& $\mathbf{N}$ & $\mathbf{\%}$ & $\mathbf{N}$ & $\mathbf{\%}$ \\
\hline Controls & 60 & 60 & 40 & $40(1.5: 1)$ \\
HCM & 76 & 76 & 24 & $24(3.1: 1)$ \\
Mean age at onset & $38.8 \pm 13.6$ yrs & & $38.4 \pm 18.1 \mathrm{yrs}$ \\
Familial & 44 & 57.8 & 16 & $66.6(1: 1.1)$ \\
Non-familial & 32 & 42.1 & 8 & $33.3(1: 1.2)$ \\
\hline
\end{tabular}

\subsection{Methodology}

Genomic DNA isolation from the blood samples was carried out following the methodology as described by Lahiri et al (1992). The isolated DNA was amplified by Polymerase chain reaction (PCR) with the respective primers. The PCR reaction was carried out in $0.2 \mathrm{ml}$ tubes with $25 \mathrm{ul}$ of master mix in each tube. Each tube containing 100ng of genomic DNA, 50pmoles each of forward and reverse primer, 100um of dNTP, 1X PCR buffer and water to make up the volume.

PCR based RFLP analysis was carried out for genotyping of $A C E-2$, and HSP70 polymorphisms, and for $T N F-\alpha$, ARMS PCR was considered. The primers used for $A C E-2$, $T N F-\alpha$ and $H S P 70$ polymorphisms were as referred by [6, 
15-16] respectively. The PCR Conditions followed were initial denaturation at $95^{\circ} \mathrm{C}$ for $5 \mathrm{~min}$, denaturation step at 950C for $1 \mathrm{~min}$, annealing at $59^{\circ} \mathrm{C}(A C E-2), 63.1^{\circ} \mathrm{C}(T N F-\alpha)$ and $55-60^{\circ} \mathrm{C}(H S P 70)$ for $1 \mathrm{~min}$, extension at $72^{\circ} \mathrm{C}$ for $1 \mathrm{~min}$ for 30 cycles and final extension was carried out at $72^{\circ} \mathrm{C}$ for 5-10 min. The PCR products were subjected to RFLP analysis with the respective restriction enzymes and were later checked on $10 \%$ native PAGE gel (Table 2 ).

Table 2. Primer sequences and restriction enzymes used for ACE2, TNF- $\alpha$ and HSP70

\begin{tabular}{|c|c|c|c|}
\hline Polymorphism & Primers & Amplimer (bp) & RFLP \\
\hline$A C E-2$ (rs 2106809) & $\begin{array}{c}\text { FP -GAAAGCCAGATGCTTTAACAAG RP - } \\
\text { TTTTTCCATATCTCTATCTGATCG }\end{array}$ & $207 \mathrm{bp}$ & Taq-1: $180+27$ \\
\hline$T N F-\alpha-308 \mathrm{G} / \mathrm{A}$ (rs 1800629) & $\begin{array}{c}\text { F.P -5'TCT CGG TTT CTT CTC CAT CG-3' R.P 'G' 5'ATA } \\
\text { GGT TTT GAG GGG CAT GG-3' R.P 'A' 5'-ATA GGT TTT } \\
\text { GAG GGG CAT GA-3' }\end{array}$ & $183 b p$ & Allele specific PCR \\
\hline $\begin{array}{l}\text { HSP-70-1 }+190 \mathrm{G} / \mathrm{C} \\
\quad(\mathrm{rs} 1043618)\end{array}$ & $\begin{array}{l}\text { F:CGCCATGGAGACCAACACCC } \\
\text { R:GCGGTTCCCTGCTCTCTGTC }\end{array}$ & $488 \mathrm{bp}$ & Bsr BI: 461+27 \\
\hline HSP-70-2 +1267A/G (rs1061581) & $\begin{array}{l}\text { F:CATCGACTTCTACACGTCCA } \\
\text { R:CAAAGTCCTTGAGTCCCAAC }\end{array}$ & $1118 \mathrm{bp}$ & Pst-1: $934+184$ \\
\hline HSP-70-hom +2437T/C (rs2227956) & $\begin{array}{c}\text { F: GTCCCTGGGGCTGGAGACGG } \\
\text { R:GATGATAGGGTTACACATCTGCT }\end{array}$ & $627 \mathrm{bp}$ & NcoI: $354+273$ \\
\hline
\end{tabular}

Table 3. Genotypic frequency distribution and Odds relative risk estimation of ACE-2 in controls and the patient group

\begin{tabular}{llllll}
\hline Allele/Genotype & Controls & & HCM & Odds Ratio (95\%CI) \\
\hline Male & $(\mathrm{n}=60)$ & $(\%)$ & $(\mathrm{n}=76)$ & $(\%)$ & \\
C & 56 & 93.3 & 73 & 96 & $0.51(0.1477-1.787)$ \\
T & 4 & 6.6 & 3 & 4 & 0.74 \\
Females & $(\mathrm{n}=40)$ & $(\%)$ & $(\mathrm{n}=24)$ & $(\%)$ & $1(0.616-1.6214)$ \\
CC & 26 & 65 & 16 & 66.6 & $1.136(0.60-2.14)$ \\
CT & 10 & 25 & 7 & 29.1 & $0.40(0.120-1.32)$ \\
TT & 4 & 10 & 1 & 4.1 & 1 \\
\hline
\end{tabular}

Table 4. Genotypic distribution and relative risk estimation of TNF- $\alpha$ and HSP70 in controls and HCM group

\begin{tabular}{|c|c|c|c|c|c|c|}
\hline Site & Genotypes & Controls $\mathrm{N}=100$ & Patients $N=100$ & Odds Ratio & CI $(95 \%)$ & P-Value \\
\hline \multirow[t]{3}{*}{$T N F-\alpha$} & $\mathrm{G} / \mathrm{G}$ & 44 & 47 & 1.00 & & \multirow{3}{*}{0.62} \\
\hline & $\mathrm{G} / \mathrm{A}$ & 38 & 40 & 0.99 & $(0.54-1.80)$ & \\
\hline & $\mathrm{A} / \mathrm{A}$ & 18 & 13 & 0.68 & $(0.30-1.54)$ & \\
\hline \multirow[t]{4}{*}{ HSP 70-1 } & $+190 \mathrm{G} / \mathrm{C}$ & & & & & \multirow{4}{*}{0.0051} \\
\hline & GG & 36 & 31 & 1.00 & -- & \\
\hline & GC & 57 & 46 & 0.94 & $0.51-1.74$ & \\
\hline & $\mathrm{CC}$ & 7 & 23 & $3.82 *$ & $1.44-10.09 *$ & \\
\hline \multirow[t]{4}{*}{ HSP70-2 } & $+1267 \mathrm{~A} / \mathrm{G}$ & & & & & \multirow{4}{*}{0.0036} \\
\hline & AA & 33 & 21 & 1.00 & -- & \\
\hline & $\mathrm{AG}$ & 51 & 43 & 1.32 & $0.67-2.62$ & \\
\hline & GG & 16 & 36 & $3.54 *$ & $1.58-7.90^{*}$ & \\
\hline \multirow[t]{4}{*}{ HSP70-hom } & $+2437 \mathrm{~T} / \mathrm{C}$ & & & & & \multirow{4}{*}{0.8} \\
\hline & $\mathrm{TT}$ & 19 & 16 & 0.87 & $0.38-1.95$ & \\
\hline & $\mathrm{TC}$ & 45 & 49 & 1.12 & $0.60-2.08$ & \\
\hline & $\mathrm{CC}$ & 36 & 35 & 1.00 & -- & \\
\hline
\end{tabular}

\subsection{Statistical and Insilico Analysis}

Genotypic frequency distribution and Odds relative risk estimation with $95 \%$ confidence intervals $(95 \% \mathrm{CI})$ were calculated for each of the polymorphic locus by using SNPSTAT software (www.bioinfo.iconcologia.net/snpstats)

Haplotype analysis and linkage disequilibrium were carried out by Haploview 4.2 software (http://www.broad.mit.edu/mpg/haploview/contact.php)

to examine the association between various polymorphisms. Gene-Gene interaction was analyzed by Multifactor Dimensionality Reduction (MDR 2.0) analysis

\section{Results}

The allelic, genotypic frequencies and Odds relative risk estimation of $A C E-2, T N F-\alpha$ and $H S P 70$ are given in Table 3 and Table 4 respectively. No significant deviation was observed in the genotypic frequencies and the Odds relative risk of estimation of $A C E-2, T N F-\alpha$ and $H S P 70$ hom polymorphisms among the patients and the control group. However, with respect to HSP70-1;+190G/C, the genotypic frequency distribution was observed to be significant with slight deviation from Hardy-Weinberg equilibrium in the controls. The ' $\mathrm{CC}$ ' genotype was found to be significantly 
increased (23\%) in the diseased group compared to only $7 \%$ in the control group with a significant odds ratio of 3.82 $(1.44 \pm 10.09)$. The genotypic frequency distribution of $+1267 \mathrm{~A} / \mathrm{G}$ HSP70-2 was also found to be significant wherein the variant ' $G G$ ' genotype was reported in $36 \%$ of the patients compared to only $16 \%$ in controls with a significant odds ratio of $3.54(1.58 \pm 7.90)$ (Table 3$)$.

The mean of the echocardiographic parameters viz left ventricular posterior wall thickness (LVPW), interventricular septal thickness (IVS), left ventricular outflow tract gradient (LVOT), and left ventricular ejection fraction (LVEF) were examined with respect to various genotypes (Table-5). ACE-2 and $T N F-\alpha$ did not reveal any difference with respect to the variant genotype. However, a significant mean of IVS $(2.11 \pm 0.42 \mathrm{~cm})$, LVOT $(58.7 \pm 35.1 \mathrm{mmHg})$ and LVEF $(70 \pm 6.18)$ parameters were observed in individuals with the variant ' $\mathrm{C}$ ' allele $(\mathrm{GC}+\mathrm{CC})$ of $H S P 70-1$ polymorphism while HSP70-2 polymorphism revealed a significant mean LVPW $(1.21 \pm 0.24 \mathrm{~cm})$, IVS $(2.04 \pm 0.47 \mathrm{~cm})$ and LVOT $(63.1 \pm 32.8 \mathrm{mmHg})$ in patients with variant ' $\mathrm{GG}$ ' genotype. The variant ' $\mathrm{CC}$ ' genotype of HSP70-hom was observed with a significant mean LVPW $(1.21 \pm 0.25 \mathrm{~cm})$ and LVOT $(57.3 \pm 31.0 \mathrm{mmHg})$, revealing a modifier effect of the variant genotypes on the phenotypic expression of the disease (Table $5)$.

Table 5. Comparison of Echocardiographic parameters with the modifier genes

\begin{tabular}{ccccc}
\hline & $\begin{array}{c}\text { Mean of } \\
\mathbf{L V W}(\mathbf{c m}) \\
\mathbf{X} \pm \text { SD }\end{array}$ & $\begin{array}{c}\text { Mean of } \\
\mathbf{I V S ( c m )} \\
\mathbf{X} \pm \text { SD }\end{array}$ & $\begin{array}{c}\text { Mean of } \\
\mathbf{L V T}(\mathbf{m m H g}) \\
\mathbf{X} \pm \text { SD }\end{array}$ & $\begin{array}{c}\text { Mean of } \\
\mathbf{L V E F}(\%) \\
\mathbf{X} \pm \text { SD }\end{array}$ \\
\hline$A C E-2$ & & & & \\
$\mathrm{C} / \mathrm{CC}$ & $1.18 \pm 0.24$ & $1.93 \pm 0.49$ & $57.9 \pm 30.2$ & $68.2 \pm 9.91$ \\
$(89)$ & & & & \\
$\mathrm{T} / \mathrm{CT} / \mathrm{TT}$ & $1.07 \pm 0.18$ & $1.64 \pm 0.499$ & $56 \pm 30.56$ & $67 \pm 12.2$ \\
$(11)$ & & & & \\
TNF- $\alpha$ & & & & \\
GG & $1.17 \pm 0.26$ & $1.86 \pm 0.47$ & $65.2 \pm 29.2$ & $70.09 \pm 9.1$ \\
GA & $1.17 \pm 0.23$ & $1.98 \pm 0.48$ & $47.3 \pm 27.4$ & $66.8 \pm 10.01$ \\
AA & $1.16 \pm 0.16$ & $1.87 \pm 0.30$ & $39.6 \pm 17.3$ & $63.6 \pm 8.21$ \\
$H S P 70-1$ & & & & \\
GG & $1.13 \pm 0.20$ & $1.8 \pm 0.46$ & $51 \pm 30.3$ & $66.4 \pm 11.5$ \\
GC & $1.19 \pm 0.23$ & $1.87 \pm 0.52$ & $57.4 \pm 23.1$ & $68.3 \pm 9.2$ \\
CC & $1.18 \pm 0.28$ & $2.11 \pm 0.42$ & $58.7 \pm 35.1$ & $70 \pm 6.18$ \\
$H S P 70-2$ & & & & \\
AA & $1.08 \pm 0.19$ & $1.87 \pm 0.41$ & $54.8 \pm 23.2$ & $69.8 \pm 9.2$ \\
AG & $1.17 \pm 0.24$ & $1.94 \pm 0.56$ & $52.5 \pm 30.6$ & $67.13 \pm 9.22$ \\
GG & $1.21 \pm 0.24$ & $2.04 \pm 0.47$ & $63.1 \pm 32.8$ & $68.2 \pm 10.17$ \\
$H S P 70-$ & & & & \\
hom & & & & \\
TT & $1.2 \pm 0.19$ & $2.16 \pm 0.35$ & $57.5 \pm 35.6$ & $71.1 \pm 4.58$ \\
TC & $1.12 \pm 0.22$ & $1.85 \pm 0.54$ & $53.2 \pm 24.3$ & $68.8 \pm 9.84$ \\
CC & $1.21 \pm 0.25$ & $1.89 \pm 0.43$ & $57.3 \pm 31.0$ & $65.8 \pm 10.03$ \\
\hline
\end{tabular}

The clinical symptoms was compared to the respective genotypes of the genes studied which revealed increased frequency of palpitations $(63.6 \%)$ and syncope $(27.2 \%)$ in patients with the variant $\mathrm{T} / \mathrm{CT} / \mathrm{TT}$ allele/genotype of $A C E-2$ gene while increased frequency of dyspnoea $(76.9 \%)$ and palpitations $(84.6 \%)$ were observed in patients with the variant 'AA' genotype of $T N F-\alpha$. The symptoms of angina
(47.8\%), syncope (32.2\%) and pre-syncope (39.1\%) were with the variant ' $\mathrm{CC}$ ' genotype of HSP70-1 and symptoms of dyspnoea (81.25), angina (43.75) and pre-syncope (62.5) in patients with 'TT' genotype of HSP70-hom were observed to be increased in HCM, indicating minor/variant alleles to have an influence on the clinical manifestation and disease progression in conjunction with other environmental factors/SNPs highlighting its modifier effect (Table 6).

Table 6. Clinical symptom estimates with respect to the ACE-2, TNFa and HSP70 genes

\begin{tabular}{cccccc}
\hline Genes & Dyspnoea & Palpitation & Angina & Syncope & $\begin{array}{c}\text { Pre- } \\
\text { syncope }\end{array}$ \\
\hline$A C E-2$ & & & & & \\
C/CC & 75 & 60 & 34 & 23.5 & 29.2 \\
T/CT/TT & 72 & 63.6 & 27.2 & 27.2 & 27.2 \\
$T N F-\alpha$ & & & & & \\
GG & 70.2 & 55.3 & 36.1 & 21.2 & 25.5 \\
GA & 80 & 60 & 37.5 & 32.5 & 35 \\
AA & 76.9 & 84.6 & 15.3 & 7.6 & 23 \\
$H S P 70-1$ & & & & & \\
GG & 80.6 & 60.8 & 25.8 & 17.3 & 29.0 \\
GC & 69.5 & 64.5 & 32.6 & 21.7 & 23.9 \\
CC & 78.2 & 58.6 & 47.8 & 32.2 & 39.1 \\
$H S P 70-2$ & & & & & \\
AA & 80.9 & 57.14 & 38.0 & 33.3 & 33.3 \\
AG & 76.7 & 67.4 & 27.9 & 20.9 & 27.9 \\
GG & 69.4 & 55.5 & 38.8 & 22.2 & 27.7 \\
HSP70- & & & & & \\
hom & & 62.5 & 43.75 & 18.75 & 62.5 \\
TT & 81.25 & 63.2 & 30.6 & 22.4 & 24.4 \\
TC & 71.4 & 57.1 & 34.2 & 28.5 & 20.0 \\
CC & 77.1 & & & &
\end{tabular}

\subsection{Statistical Analysis}

\subsubsection{Linkage Disequilibrium}

Linkage disequilibrium was estimated for 4 SNPs viz: TNF- $\alpha$ (rs1800629); HSP70-1 (rs1043618), HSP70-2 (rs 1061581); HSP70-hom (rs 2227956) of 6p21.3 region by HAPLOVIEW version 4.2 software which revealed a r2 value of 0.7 suggesting $H S P 70-1$ and $H S P 70-2$ to be in a disequilibrium (Fig 1). The haplotype frequency estimation of various genotypic combinations revealed patients with the haplotypes G-C-G-C (0.0805) and G-C-G-T (0.08) were observed to be at an increased risk to HCM. (Table 7)

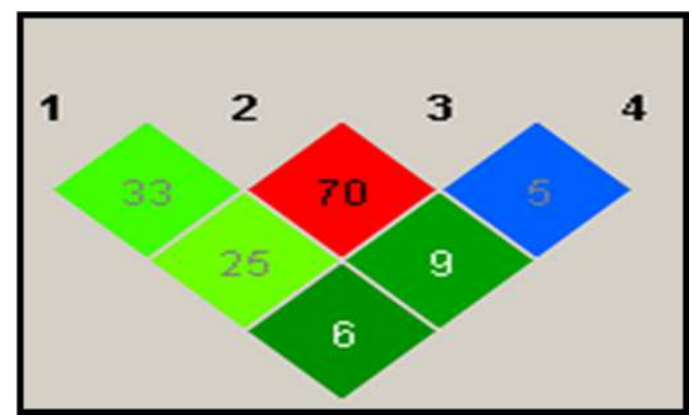

Fig 1. LD block of the modifier genes 
Table 7. Haplotype Frequency Estimation of various genotypic combinations

\begin{tabular}{ccccccccc}
\hline S.NO & TNF & HSP70.1 & HSP70.2 & HSP70.hom & Total & Controls & Patients & $\begin{array}{c}\text { Cumulative } \\
\text { frequency }\end{array}$ \\
\hline 1 & A & C & G & C & 0.2709 & 0.2797 & 0.2614 & 0.2709 \\
2 & G & G & A & T & 0.2384 & 0.2697 & 0.2064 & 0.5093 \\
3 & G & G & A & C & 0.226 & 0.25 & 0.2031 & 0.7353 \\
4 & G & C & G & C & 0.0593 & 0.0378 & 0.0805 & 0.7946 \\
5 & G & C & G & T & 0.0587 & 0.0375 & 0.08 & 0.8534 \\
6 & A & G & A & T & 0.0406 & 0.0653 & 0.0155 & 0.894 \\
7 & G & G & G & T & 0.0338 & 0.0175 & 0.05 & 0.9277 \\
8 & G & G & G & C & 0.0337 & 0.0175 & 0.05 & 0.9615 \\
9 & A & G & G & T & 0.02 & 0.025 & 0.015 & 0.9815 \\
\hline
\end{tabular}

\subsubsection{Gene-Gene Interaction by MDR Analysis}

MDR analysis was carried out for the all the 5 SNPs to establish the gene-gene interactions. The four locus model was the best interaction predicted by MDR analysis with a cross validation consistency (CVC) of 10/10 [17]. Individuals with the variant genotypes of HSP70-1 and HSP70-2 or in combination with the heterozygotes of $T N F-\alpha$ and $H S P 70$ hom and $A C E-2$ are confounded to the high risk group while patients with the wild / heterozygote genotypes at 2 or more loci belong to the low risk group (Fig 2) . This was further supported by a dendrogram which revealed a linkage disequilibrium between these 4 SNPs, however $T N F$ - $\alpha$ was observed to have a synergistic interaction with the rest of the SNPs as indicated by an orange line (Fig 3).
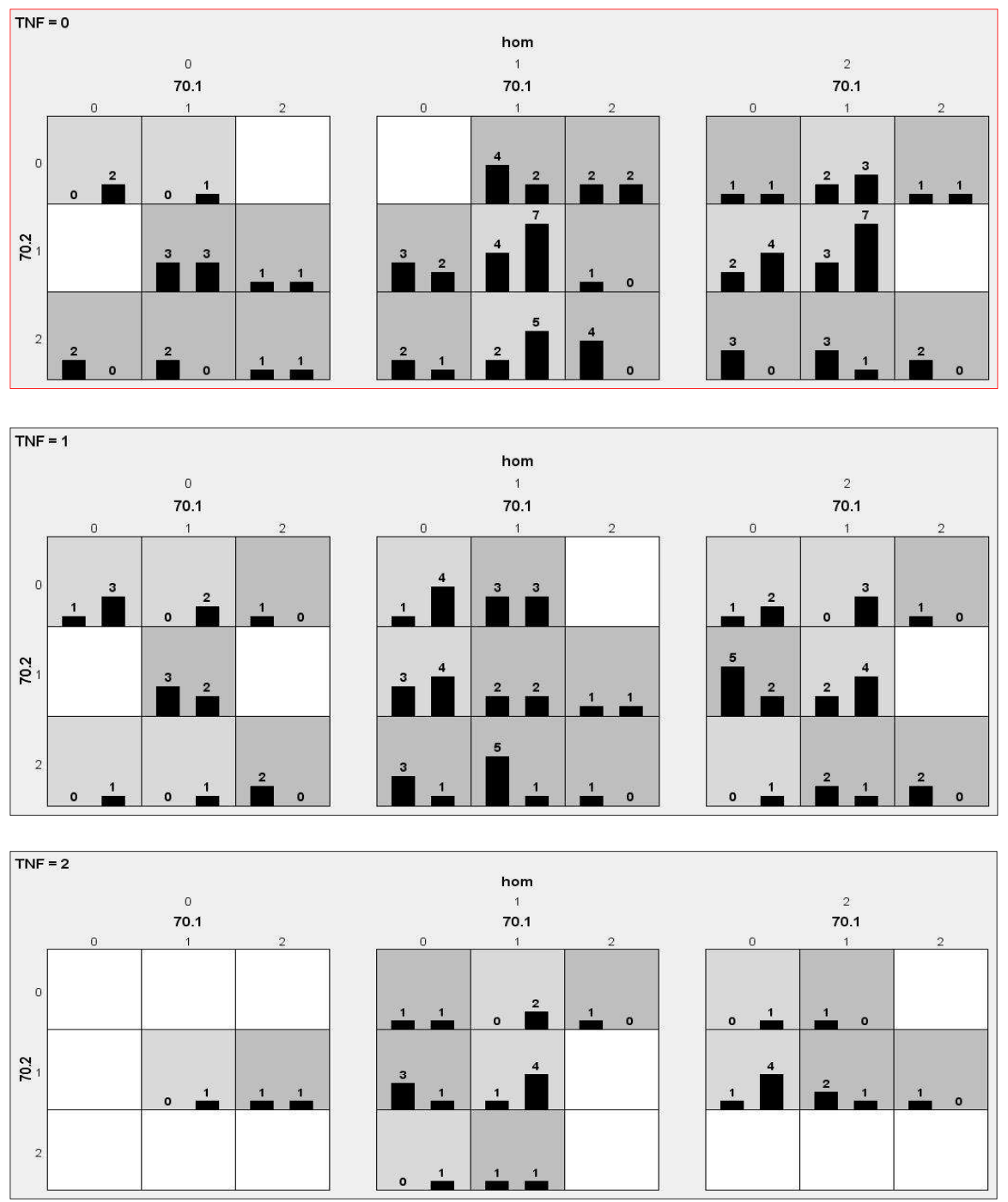

Fig 2. Graphical representation of the four-locus interaction 


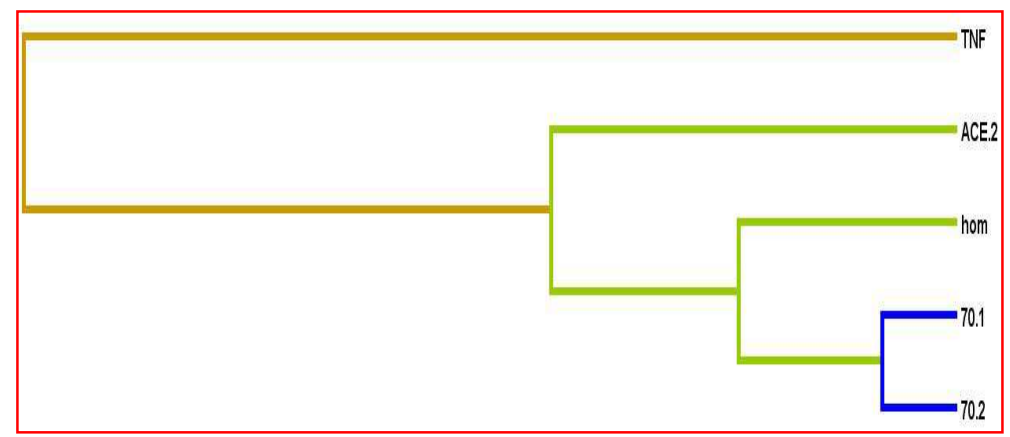

Red and orange suggests a synergistic relationship (i.e. epistasis). Yellow suggests independent effects of the SNP. Green and blue suggest redundancy or no interaction (Linkage disequilibrium).

Fig 3. Dendrogram depicting the interaction between the genes

\section{Discussion}

$\mathrm{HCM}$ is a multifactorial disease with mutations implicated in 12 sarcomeric/cytoskeletal genes. Single nucleotide polymorphisms (SNPs) in the human genome form the backbone of the inter-individual variability in susceptibility to disease and response to therapy. Accordingly, the phenotypic expression of HCM is determined not only by the causal sarcomeric gene mutations but also by the genetic backdrop of other genes as 'modifiers'. Hence SNPs of various genes implicated in cardiac growth and hypertrophy are expected to influence the phenotypic expression of cardiac hypertrophy and account for the inter-individual variation in the progression and severity of the disease [1-2]

The present study considered genotyping of 5 SNPs in 3 modifier genes viz; $A C E-2, T N F-\alpha$ and $H S P 70$. With respect to the genetic heterogeneity and ethnic variation, this is a first study to report the interaction of modifier genes in HCM patients of Indian origin. The role of rs2106809 polymorphism of $A C E-2$ gene was reported in a Chinese study wherein the minor allele was found to be associated with left ventricular hypertrophy in male patients which emphasizes the role of $A C E-2$ gene in conferring gender specific variability. Though the present study did not reveal a significant association with the patient group, increased clinical manifestations with the variant allele (T/CT/TT) were observed,which emphasize the role of $A C E-2$ gene not as a causative factor but as a modifier of the phenotype.

$T N F-\alpha$ has been largely implicated in the myocardial depression associated with septic shock as well as in the pathogenesis of heart failure [18-19]. Numerous studies have demonstrated that circulating levels of $T N F-\alpha$ are elevated in patients with congestive heart failure (CHF) and there is a significant correlation between plasma levels of $T N F-\alpha$ with severity, symptoms, and survival of the patients [20-21]. $T N F-\alpha$ mediates heart failure by a reduction in intracellular $\mathrm{Ca} 2+$, direct negative inotropic effects, interference with nitric oxide synthase, cardiomyocyte apoptosis and/or increased production of superoxide anions. However, $T N F-\alpha$ production may be secondary to heart failure and hence could be act as a modifier of HCM phenotype [22].
The present study did not reveal any significant difference in the genotypic frequency distribution and the odds relative risk estimation. However, patients with the variant ' $A$ ' allele seemed to have an influence on the onset of dyspnoea and palpitations, further enlightening on the rare allele wherein the presence of other pathogenic SNPs may modify the phenotype by influencing the transcriptional activity of the protein eventually affecting the HCM phenotype.

The HSP70 polymorphisms have been implicated in various diseases like parkinson's disease, schizophrenia, breast carcinoma, ischemic stroke, coronary artery disease etc. Studies have reported the over expression of Heat shock proteins have a cardioprotective role and genetic variants of HSP-70 would decrease its potential to protect the cells against ischemia, which ultimately may affect the ability to inhibit apoptosis resulting in cardiac hypertrophy associated HCM [23-26]. There are three HSP70 polymorphisms in the intronic region of which $H S P 70-1 ;+190 \mathrm{G} / \mathrm{C}$ polymorphism is a silent substitution which lies 26 bp upstream of the translation start site in the 5'UTR region and has been associated with various diseases like Parkinson's disease, High-altitude illness, diabetes mellitus [16]. The genotypic frequency distribution revealed a significant association of the variant ' $C$ ' allele with an odds ratio of 3.82(1.44-10.09). This was further accompanied by increased frequencies of symptoms of palpitation, angina, syncope and pre-syncope along with a significant mean of IVS $(2.11 \pm 0.42 \mathrm{~cm})$, LVOT $(58.7 \pm 35.1 \mathrm{mmHg})$ and $\operatorname{LVEF}(70 \pm 6.18 \%)$.

The HSP70-2; $+1267 \mathrm{~A} / \mathrm{G}$ polymorphism lies in the coding region of the gene. The association of variable HSP70-2 mRNA expression and the $+1267 \mathrm{~A} / \mathrm{G}$ polymorphism has been reported previously which suggested that the interindividual differences in HSP-70 expression may be related to regulatory mechanisms distinct from transcriptional regulation [27]. The present study revealed increased frequency distribution of the variant genotype with statistically significant odds ratio of 3.54(1.58-7.90). Clinically, patients with the variant genotype revealed significant difference in the echocardiographic characteristics, wherein the mean left ventricular posterior wall $(1.21 \pm 0.24 \mathrm{~cm}), \quad$ interventricular septal thickness 
$(2.04 \pm 0.47 \mathrm{~cm})$ and left ventricular outflow tract gradient $(63.1 \pm 32.8 \mathrm{mmHg})$ were found to be increased along with increased frequency of dyspnoea, palpitations and angina. Therefore ' $G$ ' allele and its encoded product may play a role in the disease severity or act as a modifier gene. Reports have shown that the polymorphic site in HSP70-2 has a functional significance wherein synonymous gene variation influences the enzyme activity and its expression, possibly by affecting the secondary RNA structure, its stability and the timing of co-translational folding which alters the substrate or inhibitor binding sites [28-29].

The HSP70hom $+2437 \mathrm{~T} / \mathrm{C}$ polymorphism results in a missense substitution Met493Thr, which may affect the substrate specificity and chaperone activity of HSP70hom protein [16]. Genotyping of HSP70-hom did not reveal any significant relative risk estimates in the patient group however, patients with the variant ' $\mathrm{C}$ ' allele revealed increased frequency of syncope and palpitations, indicating individuals with the ' $\mathrm{C}$ ' allele could be at increased risk for HCM. SNPs in the coding region like in HSP70-2 and HSP70-hom affects the affinity of the HSP70 proteins and the ATPase activity, while nucleotide changes in the flanking regions like in HSP70-1 might affect the inducibility, degree of expression or stability of mRNA.

Since both $T N F-\alpha$ and HSP70 genes are localized to the class III region of the major histocompatibility complex which is a highly polymorphic locus, it may account for the ethnic/genetic diversity with respect to various populations. Disease heterogeneity among patient groups may also lead to difficulties in the comparison of such studies and their reproducibility. Therefore a study on a larger sample size in various racial groups is warranted to emphasize its role in the pathogenesis of cardiomyopathy associated heart failure.

The interaction between the various modifier genes studied revealed that both $H S P 70-1$ and $H S P 70-2$ are in linkage disequilibrium which was further substantiated by MDR analysis revealing a redundant interaction between $A C E-2$ and $H S P 70$ polymorphisms whereas $T N F-\alpha$ has a synergistic effect. Individuals with the variant genotypes of HSP70-1 and HSP70-2 or in combination with the heterozygotes of $T N F$ - $\alpha$; HSP70 hom and $A C E-2$ are confounded to the high risk group and therefore patients with haplotypes GCGC and GCGT are at an increased risk to HCM. Hence the present study emphasizes the role of $A C E-2, T N F-\alpha$ and $H S P 70$ genes as modifiers of the HCM, in conjunction with other casual mutations/SNPs.

\section{Conclusion}

The present study is an attempt to understand the interaction between the three modifier genes; ACE-2, $T N F-\alpha$ and HSP70. Although these genes were individually studied by various groups in other diseases, this is first study to report the cumulative effect of these three genes in HCM patients and emphasize their role as Modifiers of HCM phenotype in conjunction with other causal sarcomeric mutations.

\section{Acknowledgement}

The contribution of all the authors is acknowledged in the preparation of the manuscript. We thank Indian Council of Medical Research (ICMR) and Department of Science and Technology (DST), New Delhi, India for the financial assistance.

\section{References}

[1] Maron BJ. Hypertrophic cardiomyopathy: a systematic review. JAMA, 2002. 287: p.1308-1320,

[2] Marian AJ. Modifier genes for hypertrophic cardiomyopathy. Current Opinion in Cardiology, 2002. 17: p. 242-252.

[3] Ortlepp JR., et al. Genetic polymorphisms in the reninangiotensin-aldosterone system associated with expression of left ventricular hypertrophy in hypertrophic cardiomyopathy: a study of five polymorphic genes in a family with a disease causing mutation in the myosin binding protein $\mathrm{C}$ gene. Heart, 2002. 87: p. 270-275.

[4] Donoghue M, et al. A novel angiotensin-converting enzymerelated carboxypeptidase (ACE2) converts angiotensin I to angiotensin 1-9. Circulation Research, 2000, 87: p.1-9.

[5] Danilczyk U et al. A story of two ACEs. Journal of Molecular Medicine, 2003 81: p. 227-234

[6] Wang SX, et al. Polymorphisms of angiotensinconverting enzyme 2 gene associated with magnitude of left ventricular hypertrophy in male patients with hypertrophic cardiomyopathy. Chinese Medical Journal, 2008. 121: p. 2731

[7] Yokoyama T, et al. Tumor Necrosis Factor- $\alpha$ Provokes a Hypertrophic Growth Response in Adult Cardiac Myocytes. Circulation, 1997. 95: p. 1247-1252

[8] Kapadia S, et al. Soluble TNF binding proteins modulate the negative inotropic properties of $T N F-\alpha$ in vitro. American Journal of Physiology, 1995. 268: p. 517-525

[9] Bryant D, et al. Cardiac failure in transgenic mice with myocardial expression of tumor necrosis factor- $\alpha$. Circulation, 1998. 97: p. 1375-1381.

[10] Bozkurt B, et al. Pathophysiologically relevant concentrations of tumor necrosis factor- $\alpha$ promote progressive left ventricular dysfunction and remodeling in rats. Circulation, 1998. 97: p. $1382-1391$

[11] Alfonso S and Richiardi PM A polymorphic variation in a putative regulation box of the TNFA promoter region. Immunogenetics, 1994. 39(2): p.150-154.

[12] Wilson AG, et al. Effects of a polymorphism in the human tumor necrosis factor alpha promoter on transcriptional activation. Proceedings of National Academy of Sciences U S A, 1997. 94: p. 3195-3159.

[13] Gething MJ and Sambrook J. Protein folding in the cell. Nature, 1992. 355: p. 33-45.

[14] Lahiri D and Nurnberger J. A rapid non enzymatic method for preparation of HMW DNA from blood for RFLP studies. Nucleic acids Research, 1991. 19: p. 5444 
[15] Verjans GM et al. Polymorphism of tumor necrosis factoralpha $(T N F-a)$ at position- $308 \mathrm{G} / \mathrm{A}$ in relation to ankylosing spondylitis. Clinical and Experimental Immunology, 1994. 97: p. $45-47$.

[16] Milner CM and Campbell RD. Polymorphic analysis of the three MHC-linked HSP70 genes. Immunogenetics, 1992. 36: p. $357-362$.

[17] Ritchie MD et al. Multifactor-dimensionality reduction reveals high-order interactions among estrogen-metabolism genes in sporadic breast cancer. American Journal of Human Genetics, 2001. 69(1): p. 138-147.

[18] Parrillo JE, et al. A circulating myocardial depressant substance in humans with septic shock. Journal of Clinical Investigations, 1985. 76: p.1539-1553.

[19] Michie HR, et al. Detection of circulating tumor necrosis factor after endotoxin administration. New England Journal of Medicine, 1998. 318:p. 1481-1486.

[20] Torre-Amione G, et al. Tumor necrosis factor-a and tumor necrosis factor receptors in the failing human heart. Circulation, 1996. 93: p. $704-711$.

[21] Nozaki N, et al. Soluble tumor necrosis factor receptors are elevated in relation to severity of congestive heart failure. Japanese Circulation Journal, 1997.61: p. 657-664.

[22] Patel R, et al. Variants of trophic factors and expression of cardiac hypertrophy in patients with hypertrophic cardiomyopathy. Journal of Molecular Cell, 2000. 32(12): p. 2369-2377

[23] Zhu J, et al. Increased Serum Levels of Heat Shock Protein 70 Are Associated With Low Risk Of Coronary Artery Disease. Arteriosclerosis, Thrombosis and Vascular Biolology, 2003. 23: p. 1055-1059.

[24] Yih- $\mathrm{Ru} \mathrm{Wu}$, et al. Analysis of heat-shock protein 70 gene polymorphisms and the risk of Parkinson's disease. Human Genetics, 2004.114: p. 236-241.

[25] Liu J, et al. Effects of polymorphisms of heat shock protein 70 gene on ischemic stroke, and interaction with smoking in China. Clinical Chimica Acta, 2007. 384: p. 64-68.

[26] Kim J, et al. Association analysis of heat shock protein 70 gene polymorphisms in schizophrenia. European Archives of Psychiatry Clinical Neuroscience, 2008. 258: p. 239-244.

[27] Pociot F, et al. Polymorphic analysis of the human MHClinked heat shock 70 (HSP70-2) and HSP70-hom genes in insulin-dependent diabetes mellitus (IDDM).Scandinavian Journal of Immunology, 1993.38: p.491

[28] Nackley AG, et al. Human Catechol-O-Methyltransferase Haplotypes Modulate Protein Expression by Altering mRNA Secondary Structure. Science, 2006. 314: p. 1930-1933.

[29] Kimchi-Sarfaty C, et al. A "Silent" Polymorphism in the MDR1 Gene Changes Substrate Specificity. Science, 1998. 315: p. 525-528. 